\title{
Acknowledgement to Reviewers of Games in 2015
}

\author{
Games Editorial Office \\ Published: 2 February 2016 \\ MDPI AG, Klybeckstrasse 64, CH-4057 Basel, Switzerland; games@mdpi.com
}

The editors of Games would like to express their sincere gratitude to the following reviewers for assessing manuscripts in 2015.

We greatly appreciate the contribution of expert reviewers, which is crucial to the journal's editorial decision-making process. Several steps have been taken in 2015 to thank and acknowledge reviewers. Good, timely reviews are rewarded with a discount off their next MDPI publication. By creating an account on the submission system, reviewers can access details of their past reviews, see the comments of other reviewers, and download a letter of acknowledgement for their records. In addition, MDPI has launched a collaboration with Publons, a website that seeks to publicly acknowledge reviewers on a per journal basis. This is all done, of course, within the constraints of reviewer confidentiality. Feedback from reviewers shows that most see their task as a voluntary and mostly unseen work in service to the scientific community. We are grateful to our reviewers for the contribution they make.

\begin{tabular}{lll} 
Agranov, Marina & Jacobson, Sarah & Robbett, Andrea \\
Akin, Ethan & Jakob, Michael & Rode, Ashwin \\
Alfano, Mark & Kamada, Yuichiro & Rong, Zhihai \\
Andreozzi, Luciano & Karle, Heiko & Roy, Nilanjan \\
Bach, Lars & Karos, Dominik & Rusch, Hannes \\
Beard, Rodney & Katzman, Brett & Rusinowska, Agnieszka \\
Bednarik, Peter & Kawagoe, Toshiji & Saral, Krista \\
Berger, Joël & Khadjavi, Menusch & Sasaki, Tatsuya \\
Bloembergen, Daan & Kocher, Martin & Shi, Xinyan \\
Boehnke, Jörn & Lafky, Jonathan & Simon, Jay \\
Brañas-Garza, Pablo & Laing, Tim & Skarbek, David \\
Breitmoser, Yves & Lazarova, Emiliya & Sluckin, Tim J. \\
Broom, Mark & Lessard, Sabin & Smith, Alexander \\
Capraro, Valerio & Lucas, Gale & Sofianos, Andis \\
Chapkovskiy, Philipp & Lynham, John & Song, Fei \\
Chapman, Gretchen & Mak, Vincent & Spenkuch, Jorg \\
Chatterjee, Ishita & Mallozzi, Lina & Stewart, Alexander \\
Chen, May-Ru & Martinez-VAquero, Luis & Sujarittanonta, Pacharasut \\
Christiansen, Nels Peter & Molinero, Xavier & Szabolcs, Számadó \\
Colman, Andrew & Nagatsu, Michiru & Szell, Michael \\
Courtois, Pierre & Nagel, Rosemarie & Tanimoto, Jun \\
Dasgupta, Utteeyo & Nakamura, Mitsuhiro & Tao, Yi \\
\hline
\end{tabular}




$\begin{array}{lll}\text { Davies, Elwyn } & \text { Nax, Heinrich } & \text { Thomson, William } \\ \text { de Fontenay, Catherine } & \text { Non, Arjan } & \text { Todd, Peter } \\ \text { de Oliveira, Angela } & \text { Norde, Henk } & \text { Torgler, Benno } \\ \text { Diamantaras, Dimitrios } & \text { Normann, Hans-theo } & \text { Tsiropoulou, Eirini Eleni } \\ \text { Dittrich, Marcus } & \text { Nosenzo, Daniele } & \text { Uchida, Satoshi } \\ \text { dos Santos, Miguel } & \text { Onderstal, Sander } & \text { Van Der Ploeg, Rick } \\ \text { Drouvelis, Michalis } & \text { Østerdal, Lars Peter Raahave } & \text { Van Veelen, Matthijs } \\ \text { Dulleck, Uwe } & \text { Pavlov, Valery } & \text { Varkey, Paul } \\ \text { Friedman, Willa } & \text { Peeters, Ronald } & \text { Veszteg, Róbert Ferenc } \\ \text { García, Julián } & \text { Peleg, Bezalel } & \text { Vyrastekova, Jana } \\ \text { Gershman, Samuel } & \text { Perc, Matjaž } & \text { Weikard, Hans-Peter } \\ \text { Grinberg, Maurice } & \text { Perez-Richet, Eduardo } & \text { Weirich, Paul } \\ \text { Grujic, Jelena } & \text { Ploner, Matteo } & \text { Wolff, Irenaeus } \\ \text { Gürerk, Özgür } & \text { Polonio, Luca } & \text { Young, Michael } \\ \text { Harman, Jason } & \text { Prato, Carlo } & \text { Zápal, Jan } \\ \text { Heinrich, Timo } & \text { Pusillo, Lucia } & \text { Zapechelnyuk, Andriy } \\ \text { Hindersin, Laura } & \text { Quérou, Nicolas } & \text { Zhang, Qingpeng } \\ \text { Hinsz, Verlin } & \text { Raí, Birendra } & \text { Zudenkova, Galina } \\ \text { Hofmann, Eva } & \text { Rand, David } & \text { Zultan, Ro'i } \\ \text { Hummel, Patrick } & \text { Rauhut, Heiko } & \\ \text { Imhof, Lorens } & \text { Ray, Indrajit } & \\ & & \\ & & \end{array}$

(C) 2016 by the author; licensee MDPI, Basel, Switzerland. This article is an open access article distributed under the terms and conditions of the Creative Commons by Attribution (CC-BY) license (http://creativecommons.org/licenses/by/4.0/). 